\title{
THE ORDER DUAL OF AN ABELIAN VON NEUMANN ALGEBRA
}

\author{
PETER G. DODDS
}

(Received 20 March 1972)

Communicated by E. Strzelecki

\section{Introduction}

The usual technique for dealing with an abelian $W^{*}$-algebra is to consider it, via the Gelfand theory, as the algebra of all continuous complex-valued functions on an extremally disconnected compact Hausdorff space with a separating family of normal linear functionals. An alternative approach, outlined in [2] and [10], is to develop the theory within the framework of Riesz spaces (linear vector lattices) where the order properties of the self-adjoint operators play an important and natural role. It has been known for a long time that the self-adjoint part of an abelian $W^{*}$-algebra is a Dedekind complete Riesz space under the natural ordering of self-adjoint operators, but it is only relatively recently that a proof of this fact has been given that is independent of the Gelfand theory, and the interested reader may consult [2] or [10] for the details. This approach is essentially foreshadowed in [6] and provides a very satisfying introduction to the theory of commutative rings of operators. From this point of view, the spectral theorem for self-adjoint operators falls naturally into place as an easy consequence of the spectral theorem of $H$. Freudenthal. In this paper, the line of approach via Riesz spaces is developed further and several well known results are shown to follow as elementary consequences of the order structure of the algebra.

The author would like to express his gratitude to Professor W. A. J. Luxemburg for many helpful suggestions relating to the subject matter of this paper.

\section{Preliminary information}

We shall adhere to the notation and terminology from the theory of Riesz spaces as developed in [2], [3] and [4]. If $L$ is a Riesz space, we write $f^{+}=f \vee o$, $f^{-}=(-f) \vee o,|f|=f \vee(-f)$, from which $f=f^{+}-f^{-},|f|=f^{+}+f^{-}$for 
every $f \in L$. Elements $f, g \in L$ are called disjoint if $|f| \wedge|g|=0$ and this is denoted by $f \perp g$. If $D$ is an arbitrary subset of a Riesz space $L$, we set $D^{d}=\{f \in L: f \perp D\}$. The Riesz space $L$ will be called Dedekind complete if every non-empty subset of $L$ which is bounded above has a least upper bound. A Riesz subspace $K$ of a Riesz space $L$ is called an order ideal whenever, given $f \in K, g \in L$ with $0 \leqq|g| \leqq|f|$, it follows that $g \in K$. An order ideal $K$ is called a band whenever it follows from $0 \leqq f_{\tau} \uparrow \tau, f_{\tau} \in K$ for each $\tau$, that $f \in K$. If $D$ is an arbitrary subset of $L,\langle D\rangle$ (respectively $\{D\}$ ) will denote the order ideal (respectively band) generated by $D$. If $K$ is a band if a Dedekind complete Riesz space $L$, then $L=K \oplus K^{d}$.

The real linear functional $\phi$ on the Riesz space $L$ is called order bounded if for each $0 \leqq u \in L$, the number $\sup (|\phi(f)|:|f| \leqq u)$ is finite. The set of all order bounded linear functionals is denoted by $L^{\sim}$. Under the natural definitions, $L^{\sim}$ is always a Dedekind complete Riesz space. The functional $\phi \in L^{\sim}$ is said to be an integral if $0 \leqq u_{n} \downarrow 0$ implies $\phi\left(u_{n}\right) \rightarrow 0$ as $n \rightarrow \infty$. The collection of all integrals is a band in $L^{\sim}$ and is denoted by $L_{c}^{\sim}$. The element $\phi \in L^{\sim}$ is called a singular functional if $\phi \perp \psi$ for all $\psi \in \tilde{L_{c}}$. We have $L^{\sim}=\tilde{L_{c}} \oplus \tilde{L_{s}}$ A functional $\phi \in L^{\sim}$ is called a normal integral if $u_{\tau} \downarrow_{\tau} 0 \operatorname{implies}_{\inf _{\tau}}\left|\phi\left(u_{\tau}\right)\right|=0$. The set of normal integrals is a band in $\tilde{L_{c}}$ and we set $\tilde{L_{c}}=\tilde{L_{c, s n}} \oplus \tilde{L_{n}}$. For any $\phi \in L^{\sim}$, set $N_{\phi}=\left\{f \in L:|\phi|\left(\mid f_{/}\right)=0\right\}, N_{\phi}$ is an order ideal in $L$. If $L$ is Dedekind complete, then $\phi \in \widetilde{L_{n}}$ iff $N_{\phi}$ is a band. If $L$ is Dedekind complete and $0 \leqq \phi, \psi \in \widetilde{L_{n}}$, then $\{\psi\} \subseteq\{\phi\}$ iff $N_{\phi} \subseteq N_{\psi}$ and in this case $\psi=\sup _{n}(n \phi \wedge \psi)$.

\section{Integrals which are singular normal}

We take as our starting point an abelian $W^{*}$-algebra $M$ of operators acting in a complex Hilbert space $\mathscr{H}$. Re $M$ shall denote the real linear space of selfadjoint elements of $M$; with the natural definition of partial order, $\operatorname{Re} M$ is a Dedekind complete Riesz space with a separating family of normal integrals. The operator norm is a Riesz norm on $\operatorname{Re} M$, under which $\operatorname{Re} M$ is an abstract $L_{\infty}$-space. Moreover if $A_{\tau} \uparrow_{\tau} A$ in $\operatorname{Re} M$, then the system $A_{\tau}$ converges to $A$ in the strong operator topology. A linear functional $\phi \in M^{*}$ (Banach dual) is called self-adjoint if for each $T \in M, \phi\left(T^{*}\right)=\overline{\phi(T)}$. The collection of all such $\phi$ will be denoted by $\operatorname{Re}\left(M^{*}\right)$. Denote by $M_{n}$ the closed subspace of $M^{*}$ which consists of those linear functionals which are continuous in the ultraweak topology of operators, and set $(\operatorname{Re} M)_{n}=M_{n} \cap \operatorname{Re}\left(M^{*}\right)$. It is easy to show that $\operatorname{Re}\left(M^{*}\right)$ $=(\operatorname{Re} M)^{*}=(\operatorname{Re} M)^{\sim}$ and that $\operatorname{Re}\left(M_{n}\right)=(\operatorname{Re} M)_{n}^{\sim}$ and accordingly the proofs are omitted. In this section, we examine $(\operatorname{Re} M)_{c, s n}^{\sim}$ and ask whether every integral on $\operatorname{Re} M$ is in fact normal. It is clear that only positive functionals need be considered. If $0 \leqq \phi \in(\operatorname{Re} M)^{\sim}$, it is well known that $\phi$ is a normal integral iff for each family $\left\{E_{i}\right\}$ of pairwise disjoint projections of $M$, it follows that $\phi\left(\Sigma E_{i}\right)=\Sigma \phi\left(E_{i}\right)$. From this it is clear that if every family of pairwise disjoint 
projections of $M$ is at most countable, then the notions of integral and normal integral coincide. In particular, if $\mathscr{H}$ is separable then every integral is normal.

Suppose now that $P$ is any projection of $M$. A decomposition of $P$ is a collection $\left\{P_{\alpha}\right\}_{\alpha \in \mathscr{A}}$ of pairwise disjoint projections of $M$ which satisfies $\sup _{\alpha} P_{\alpha}=P$. The cardinal of the index set $\mathscr{A}$ is called the cardinal of the decomposition. Recall that a set $X$ is said to have a measurable cardinal if there exists a countablyadditive measure $v$ on the collection of all subsets of $X$ such that $v(X)=1$, and $v(F)=0$ for every finite subset $F$ of $X$. If such a measure $v$ does not exist, then $X$ is said to have non-measurable cardinal.

THEOREM 3.1. Let $\phi \geqq 0$ be an integral on $\operatorname{Re} M$, and assume that every decomposition of the identity of $M$ has non-measurable cardinal. Then $\phi$ is a normal integral.

Proof. Let $\mathscr{B}$ denote the family of all collections $\left\{P_{\beta}\right\}$ of pairwise disjoint projections $P_{\beta}$ such that $\phi\left(P_{\beta}\right)=0$. $\mathscr{B}$ is inductively ordered by inclusion, so there is a maximal such collection $\left\{P_{\alpha}\right\}_{\alpha \in \mathscr{\alpha}}$ say. Let $P=\sup P_{\alpha}$; then $\phi(P)=0$. If not, for $A \in 2^{\alpha}$ set $v(A)=\phi\left(\sup P_{\alpha}: \alpha \in A\right)$, and observe that $\phi(P) \neq 0$ contradicts the hypothesis that $\mathscr{A}$ has non-measurable cardinal. It now suffices to show that the null ideal of $\phi, N_{\phi}$, coincides with $\{P\}$, the band generated by $P$ in $\operatorname{Re} M$. Observe that $T \in\{P\}$ iff there exists a positive integer $k$ with $|T| \leqq k P$, so that $\{P\} \subseteq N_{\phi}$. Suppose $T \in N_{\phi}$. By the spectral theorem there exists a sequence $S_{n}=\sum_{i=1}^{m_{n}} \alpha_{i} Q_{i}^{(n)}$ in $\operatorname{Re} M$, with $\alpha_{i}>0, Q_{i}^{(n)}$ projections in $M$ such that $S_{n} \uparrow|T|$ in $\operatorname{Re} M$. It follows that $\phi\left(Q_{i}^{(n)}\right)=0$ for each $i, n$. Thus $Q_{i}^{(n)} \in\{P\}$ by maximality of the system $\left\{P_{x}\right\}$, so that $S_{n} \in\{P\}$ for each $n$; hence $|T| \in\{P\}$ since $\{P\}$ is a band. Hence $\{P\}=N_{\phi}$ and $\phi$ is normal.

We note that the above result is a consequence of [5], but that the proof presented here is essentially quite simple. In passing, we observe that Theorem 3.1 answers a question concerning $\sigma$-states of von Neumann algebras raised by R. J. Plymen in [8], since a state of a von Neumann algebra is normal iff its restriction to each maximal abelian subalgebra is normal.

\section{Singular functionals}

Following the discussion of the preceding section, we will write

$$
(\operatorname{Re} M)^{\sim}=(\operatorname{Re} M)_{s n}^{\sim} \oplus(\operatorname{Re} M)_{n}^{\sim} \text {. }
$$

LeMmA 4.1. (i) If $\phi \in(\operatorname{Re} M)_{s, n}^{\sim}$, then $N_{\phi}^{d}=\{0\}$.

(ii) Let $(\operatorname{Re} M)^{a}=\left\{T \in \operatorname{Re} M:|T| \geqq S_{n} \downarrow 0 \Rightarrow\left\|S_{n}\right\| \downarrow 0\right\}$ and let $(\operatorname{Re} M)^{a n}$ $=\left\{T \in \operatorname{Re} M:|T| \geqq S_{\tau} \downarrow 0 \Rightarrow\left\|S_{\tau}\right\| \downarrow 0\right\}$. Then $(\operatorname{Re} M)^{a}=(\operatorname{Re} M)^{a n}={ }^{\perp}\left[(\operatorname{Re} M)_{s n}\right]$. Consequently, $(\operatorname{Re} M)^{a}$ is an order ideal in $\operatorname{Re} M$. 
Proof. The proof of the lemma is contained in [4] Theorem 50.4 and Theorem 53.7 (ii) of Note XV.

Definition 4.2. A non-zero projection $P$ of $M$ will be called an atom if, for any projection $Q$ of $M, 0 \leqq Q \leqq P$ implies either $Q=0$ or $Q=P$.

Lemma 4.3. Let $P$ be an atom of $M$; then $P \in(\operatorname{Re} M)^{a n}$.

Proof. If $S \in \operatorname{Re} M$ satisfies $0 \leqq S \leqq P$, then $S=\lambda P$ for some real $\lambda, 0 \leqq \lambda \leqq 1$. Since $\lambda_{n} P \downarrow 0$ iff $\lambda_{n} \downarrow 0$, it follows that $\lambda_{n} P \downarrow O$ for $0 \leqq \lambda_{n} \leqq 1$ implies $\left\|\lambda_{n} P\right\| \downarrow 0$.

TheOREM 4.4. (i) Let $P \in(\operatorname{Re} M)^{a n}$ be a projection; then $P=\sum_{i=1}^{n} P_{i}$ where the $P_{i}$ are disjoint atoms.

(ii) $T \in(\operatorname{Re} M)^{a n}$ if and only if $T=\sum_{i=1}^{\infty} \lambda_{i} P_{i}$, where the $P_{i}, i=1,2, \cdots$, are disjoint atoms and $\left|\lambda_{i}\right| \rightarrow 0$ as $i \rightarrow \infty$.

Proof. (i) Let $P \in(\operatorname{Re} M)^{a n}$ be a projection. Assume that $P$ does not dominate a single atom. By an inductive argument, there exists a sequence $\left\{Q_{n}\right\}$ of pairwise disjoint non-zero projections majorized by $P$. Observe that $P \geqq F_{m}$ $=\vee_{n \geqq m} Q_{n} \downarrow_{m} 0$. Since $\left\|F_{m}\right\|=1$ for each $m$, this contradicts the fact that $P \in(\operatorname{Re} M)^{a n}$. It follows that there exists an atom $P_{1}$ with $0 \neq P_{1} \leqq P$. If $P-P_{1} \neq 0$, there exists an atom $P_{2}$ satisfying $0 \neq P_{2} \leqq P-P_{1}$. By the above argument, this procedure breaks off after a finite number of steps, and (i) follows.

(ii) Let $T=\sum_{i=1}^{\infty} \lambda_{i} P_{i}$, where the $P_{i}$ are pairwise disjoint atoms and $\left|\lambda_{i}\right| \rightarrow 0$ as $i \rightarrow \infty$. If $0 \leqq S \leqq|T|$, then $S=\sum_{i=1}^{\infty} s_{i} P_{i}$ with $0 \leqq s_{i} \leqq\left|\lambda_{i}\right|$, and $\|S\|=\sup s_{i}$. It follows readily that $T \in(\operatorname{Re} M)^{a n}=(\operatorname{Re} M)^{a}$. Conversely, assume $0 \leqq T \in(\operatorname{Re} M)^{a n}$. By the spectral theorem and (i) it follows that $T=\sum_{i=1}^{\infty} \lambda_{i} P_{i}$, where $0 \leqq \lambda_{i} \leqq\|T\|$, and each $P_{i}, i=1,2, \cdots$, is an atom. To show that $\lambda_{i} \rightarrow 0$ as $i \rightarrow \infty$, assume $\overline{\lim }_{i} \lambda_{i}>0$; choosing a subsequence if necessary we may assume that for some $\delta>0, \lambda_{i} \geqq \delta>0$ for $i=1,2, \cdots$. Set $Q=\sum_{i=1}^{\infty} P_{i}$. Let $N$ denote the positive integers, $\beta N$ the Stone-Čech compactification of $N$ and choose $\alpha \in \beta N-N$. For each $S \in \operatorname{Re} M$ denote by $f_{S}$ the element of $l_{\infty}(N)$ defined by $f_{S}(n)=\left(S x_{n}, x_{n}\right) n=1,2, \cdots$, where $x_{n} \in \mathscr{H}$ satisfies $P_{n} x_{n}=x_{n},\left\|x_{n}\right\|=1$. Denote by $\hat{f}_{S}$ the extension of $f_{S}$ to a continuous function on $\beta N$. Define $0 \leqq \phi \in(\operatorname{Re} M)^{*}$, via $\phi(S)=\hat{f}_{S}(\alpha)$ for each $S \in \operatorname{Re} M$. It is clear that $\phi(T) \geqq \delta>0$. Since $T \in(\operatorname{Re} M)^{a}=(\operatorname{Re} M)^{a n}$, it is sufficient to show that $\phi \in(\operatorname{Re} M)_{s, n}$. Observe that $\phi(Q)=1=\phi(I)$ so that $\phi(I-Q)=0$. Write $\phi=\phi_{s}+\phi_{n}$ where $0 \leqq \phi_{s} \in(\operatorname{Re} M)_{s, n}^{\sim}, 0 \leqq \phi_{n} \in(\operatorname{Re} M)_{n}^{\sim}$. Since the $P_{i}$ are atoms and by the definition of $\phi, 0=\phi\left(P_{1}+\cdots+P_{k}\right)=\phi_{s}\left(P_{1}+\cdots+P_{k}\right)$ $+\phi_{n}\left(P_{1}+\cdots+P_{k}\right)=\phi_{n}\left(P_{1}+\cdots+P_{k}\right)$ for $k=1,2, \cdots$. Thus $\phi_{n}(Q)=0$; also $0=\phi(I-Q)=\phi_{s}(I-Q)$. Hence $\phi_{n}(I-Q)=0$ so that $\left\|\phi_{n}\right\|=\phi_{n}(I)=0$. Hence $\phi \in(\operatorname{Re} M)_{s, n}^{\sim}$ and the proof is complete. 
We note that the proof of (ii) above shows that if the dimension of $M$ is not finite, there exist non-zero elements of $(\operatorname{Re} M)_{s, n}^{\sim}$. In fact, let $\left\{P_{i}\right\}, i=1,2, \cdots$, be a system of pairwise disjoint non-zero projections of $M$. Let $\phi \in(\operatorname{Re} M)^{*}$ be defined via the $P_{i}$ as in the proof of (ii) above. Let $F_{m}=\vee_{n \geqq m} P_{n}$. Observe that $\phi\left(F_{m}\right)=1$ for each $m$. Since $F_{m} \downarrow_{m} 0$, it follows that $\phi \notin(\operatorname{Re} M)_{n}^{\sim}$. so that the singular part of $\phi$ is non-zero.

Let $A$ denote the set of atoms in $\operatorname{Re} M$. In general, the ideal generated by the atoms of $\operatorname{Re} M$ is not equal to $(\operatorname{Re} M)^{a}$. However, we do have

Corollary 4.5. $\{A\}=\left\{(\operatorname{Re} M)^{a n}\right\}$.

Proof. $\{A\} \subseteq\left\{(\operatorname{Re} M)^{a n}\right\}$ trivially. On the other hand $A^{d} \subseteq(\operatorname{Re} M)^{a d}$ by the above theorem. Therefore $\{A\}=A^{d d} \supseteq(\operatorname{Re} M)^{a} d d=\left\{(\operatorname{Re} M)^{a}\right\}$.

We may write $\operatorname{Re} M=\{A\} \oplus\{A\}^{d}$. $\{A\}$ will be called the atomic part of $\operatorname{Re} M,\{A\}^{d}$ the non-atomic part.

THEOREM 4.6. (i) If $N$ is a band in $\operatorname{Re} M$, then $N$ is a maximal band iff there exists an atom $P$ in $\operatorname{Re} M$ such that $N=\{P\}^{d}$.

(ii) $\{A\}^{d}=\cap(N: N$ is a maximal band $)$

(iii) $\{A\}=\operatorname{Re} M$ iff $(\operatorname{Re} M)_{s . n}^{\sim}=\left[(\operatorname{Re} M)^{a}\right]^{\perp}$.

Proof. (i), (ii) follow exactly as in [2], p. 57.

(iii) If $(\operatorname{Re} M)_{s . n}^{\sim}=\left[(\operatorname{Re} M)^{a}\right]^{\perp}$, let $P \in\{A\}^{d}$ be a non-zero projection. Choose $x \in \mathscr{H}$ such that $P x=x$ and consider the normal integral $\phi(\cdot)=(\cdot x, x)$. Since $\phi \in A^{\perp}$ it follows by normality that $\phi \in\{A\}^{\perp}$. In particular, $\phi \in\left[(\operatorname{Re} M)^{a}\right]^{\perp}$ so that $\phi=0$. It follows that $\{A\}^{d}=\{0\}$, hence $\{A\}=\operatorname{Re} M$.

Conversely, assume $\{A\}=\operatorname{Re} M=\left\{(\operatorname{Re} M)^{a}\right\}$. Observe that $(\operatorname{Re} M)_{s, n}^{\sim} \subseteq$ $\left([\operatorname{Re} M)^{a}\right]^{\perp}$. Assume $0 \leqq \phi$ satisfies $\phi(T)=0$ for all $T \in(\operatorname{Re} M)^{a}$. Write $\phi=\phi_{s}+\phi_{n}$ where $0 \leqq \phi_{s} \in(\operatorname{Re} M)_{s n}^{\sim}, 0 \leqq \phi_{n} \in(\operatorname{Re} M)_{n}^{\sim}$. Since $\phi_{s}$ vanishes on $(\operatorname{Re} M)^{a}$ so also does $\phi_{n}$. By normality and the assumption that $\operatorname{Re} M=$ $\left\{(\operatorname{Re} M)^{a}\right\}$, it follows that $\phi_{n}=0$. Thus $\phi=\phi_{s}$ and the proof is complete.

We conclude this section with some remarks on the case $M$ is finite dimensional.

THEOREM 4.7. The following conditions on $M$ are equivalent:

(i) $\operatorname{Re} M=(\operatorname{Re} M)^{a n}$.

(ii) $M$ is finite-dimensional.

(iii) $M$ is a reflexive Banach space.

(iv) $(\operatorname{Re} M)^{\sim}=(\operatorname{Re} M)_{n}^{\sim}$.

Proof. (i) $\Rightarrow$ (iv) and (ii) $\Rightarrow$ (iii) are obvious.

(iv) $\Rightarrow$ (i). Since the only singular functional on $\operatorname{Re} M$ is the zero functional, $\operatorname{Re} M={ }^{\perp}\left[(\operatorname{Re} M)_{s, n}^{\sim}\right]=(\operatorname{Re} M)^{a n}$. 
(iv) $\Rightarrow$ (ii). It has been noted above, that if the dimension of $M$ is not finite, then there exist non-zero elements of $(\operatorname{Re} M)_{s, n}^{\sim}$.

(iii) $\Rightarrow$ (iv). If $M$ is reflexive, assume $M_{n}^{*} \neq M^{*}$. By reflexivity of $M$ and the Hahn-Banach theorem, there exists $0 \neq T \subseteq \operatorname{Re} M$ with $T \in{ }^{\perp} M_{n}^{*}$. In particular, for each $x, y \in \mathscr{H},(T x, y)=0$. Hence $T=0$ which is a contradiction. \#

\section{The band of normal integrals}

That the normal integrals play a key role is clear from the fact that $\operatorname{Re} M$ is precisely the (Banach) dual of the band of normal integrals on Re $M$. We show that this follows from the fact that $\operatorname{Re} M$ is a perfect Riesz space. If $x \in \mathscr{H}$, we will denote by $\omega_{x}$ the normal integral given by $T \rightarrow(T x, x)$ for $T \in \operatorname{Re} M$.

For $T \in \operatorname{Re} M, \phi \in(\operatorname{Re} M)_{n}^{\sim}$, set $v(T)(\phi)=\phi(T)$. It is clear that $v: \operatorname{Re} M \rightarrow(\operatorname{Re} M)_{n}^{\sim} \sim{ }_{n}$ is linear, one to one and preserves partial order. Since the Dedekind complete Riesz space $\operatorname{Re} M$ has a separating family of normal integrals, it follows from [3] Theorem 28.4, Note VIII, and the uniform boundedness principle that $v(\operatorname{Re} M)=(\operatorname{Re} M)_{n}^{\sim} \sim{ }_{n}^{\sim}$. In the terminology of [3], $\operatorname{Re} M$ is a perfect Riesz space. Further the mapping $v$ is an isometry, which follows by observing that, for $T \in \operatorname{Re} M$,

$$
\begin{aligned}
\|T\| & =\sup \{|(T x, x)|: x \in \mathscr{H},\|x\| \leqq 1\} \\
& \leqq \sup \left\{\left|v(T)\left(\omega_{x}\right)\right|:\left\|\omega_{x}\right\| \leqq 1\right\} \\
& \leqq \sup \left\{|v(T)(\phi)|: \phi \in(\operatorname{Re} M)_{n} ;\|\phi\| \leqq 1\right\} \\
& \leqq \sup \left\{|\phi(T)|: \phi \in(\operatorname{Re} M)^{*},\|\phi\| \leqq 1\right\}=\|T\| .
\end{aligned}
$$

We will identify $\operatorname{Re} M$ with its image in $(\operatorname{Re} M)_{n}^{\sim} \sim{ }_{n}$ under the mapping $v$. These remarks establish the first part of

THEOREM 5.1. (i) $\operatorname{Re} M=(\operatorname{Re} M)_{n}^{\sim} \sim$.

(ii) $\operatorname{Re} M=(\operatorname{Re} M)_{n}^{\sim *}$.

Proof of (ii). Observe that the norm of the Banach space $(\operatorname{Re} M)_{n}^{\sim}$ is a Riesz norm which is additive on the positive cone of $(\operatorname{Re} M)_{n}^{\sim}$ and so $(\operatorname{Re} M)_{n}^{\sim}$ is an abstract $L$-space. It is well known that every bounded linear functional on an abstract $L$-space is a normal integral so that

$$
(\operatorname{Re} M)_{n,}^{\sim}{ }^{*}=(\operatorname{Re} M)_{n}^{\sim} \sim \mathcal{\sim}=(\operatorname{Re} M)_{n, n}^{\sim \sim} \sim \operatorname{Re} M .
$$

It has been shown by R. Pallu de la Barrière [7] that every normal integral on $\operatorname{Re} M$ is of the form $T \rightarrow(T x, x)$ for some $x \in \mathscr{H}$. We now proceed to an elementary (i.e. non measure-theoretic) proof of this result.

It has been noted earlier that if $0 \leqq \phi \in(\operatorname{Re} M)_{n}^{\sim}$, then $N_{\phi}$ is a band in $\operatorname{Re} M$. Writing $\operatorname{Re} M=N_{\phi} \oplus N_{\phi}^{d}$, denote by $E_{\phi}$ the component of the identity in the band $N_{\phi}^{d}$. The projection $E_{\phi}$ will be called the carrier projection of $\phi$. It is easily 
shown that if $\phi=\omega_{x}$ for some $x \in \mathscr{H}$, then $E_{\phi}=E_{x}^{M^{\prime}}$ where $M^{\prime}$ denotes the commutant of $M$, and $E_{x}^{M^{\prime}}$ denotes the orthogonal projection (in $M$ ) onto the closure in $\mathscr{H}$ of the linear subspace $\left\{M^{\prime} x\right\}$. It follows from some earlier remarks that if $0 \leqq \phi, \psi \in(\operatorname{Re} M)_{n}^{\sim}$, then $\psi \in\{\phi\}$ iff $E_{\psi} \leqq E_{\phi}$.

The next lemma is well known (see for example [9]).

LEMMA 5.2. Let $\phi, \psi$ be positive normal integrals on $\operatorname{Re} M$ which satisfy $0 \leqq \psi \leqq \phi$. There exists $H \in \operatorname{Re} M$ with $0 \leqq H \leqq I$ such that $\psi(T)=\phi(T H)$ for all $T \in M$.

If $y, z \in \mathscr{H}$, we shall write $\omega_{y} \sim \omega_{z}$ if $(T y, y)=(T z, z)$ holds for each $T \in M$. $E_{z}^{M}$ will denote the projection (in $M^{\prime}$ ) onto the closure of the linear subspace $\{M z\}$ in $\mathscr{H}$.

Lemma 5.3. Let $x \in \mathscr{H}$. Then

$$
E_{x}^{M^{\prime}}=\sup \left\{E_{z}^{M}: \omega_{z} \sim \omega_{x}\right\}
$$

where the supremum is taken in the complete lattice of projections of the $W^{*}$-algebra $M^{\prime}$.

Proof. If $z \in \mathscr{H}$ satisfies $\omega_{z} \sim \omega_{x}$, then $E_{z}^{M^{\prime}}=E_{x}^{M^{\prime}}$. Since $\boldsymbol{M}$ is abelian, $E_{z}^{M} \leqq E_{z}^{M^{\prime}}=E_{x}^{M^{\prime}}$ so that $\sup \left\{E_{x}^{M}: \omega_{z} \sim \omega_{x}\right\} \leqq E_{x}^{M^{\prime}}$. On the other hand, let $F$ be any projection of $M^{\prime}$ which satisfies $F \geqq E_{z}^{M}$ for each $z \in \mathscr{H}$ with $\omega_{z} \sim \omega_{x}$. For each unitary operator $\mathscr{B} \in M^{\prime}, \omega_{U x} \sim \omega_{x}$. Thus $F U x=U x$ for each unitary $U \in M^{\prime}$ so $E_{x}^{M^{\prime}} \leqq F$ and the lemma follows.

We now describe the band of normal integrals on $\operatorname{Re} M$.

THEOREM 5.4. (R. Pallu de la Barrière) Let $\phi$ be a positive normal integral on $\operatorname{Re} M$. There exists $y \in \mathscr{H}$ such that $\phi=\omega_{y}$.

PROOF. Let $E_{\phi}$ be the carrier projection of $\phi$. By [1] p. 19, there exists $x \in \mathscr{H}$ such that $E_{x}^{M^{\prime}}=E_{\phi}$. It follows that $\phi$ belongs to the band generated by $\omega_{x}$ in $(\operatorname{Re} M)_{n}^{\sim}$ so that $\phi=\sup \left(\phi \wedge n \omega_{x}\right), n=1,2, \cdots$. Note that the sequence $\psi_{n}=\phi \wedge n \omega_{x}$ has the following properties: (i) $\phi_{n} \uparrow n$, (ii) $0 \leqq \phi_{n} \leqq n \omega_{x}$, (iii) for each $0 \leqq T \in M, \psi(T)=\lim _{n \rightarrow \infty} \phi_{n}(T)$. By lemma 5.2, there exists a sequence $\left\{H_{n}\right\}$ of positive operators of $M$, which we may assume satisfy $H_{n}\left(I-E_{x}^{M^{\prime}}\right)=0$, such that $\phi_{n}(T)=\left(T H_{n} x, H_{n} x\right)$ for each $T \in M$ and each $n=1,2, \cdots$. Let $z \in \mathscr{H}$ satisfy $\omega_{z} \sim \omega_{x}$. Then also $\phi_{n}(T)=\left(T H_{n} z, H_{n} z\right)$. Since $\phi_{n} \uparrow n$ it follows that $H_{n} E_{z}^{M} \geqq H_{m} E_{z}^{M}$ for $n \geqq m$, so that by lemma 5.3 $H_{n} E_{x}^{M^{\prime}} \geqq H_{m} E_{x}^{M^{\prime}}$ for $n \geqq m$. Thus $H_{n} \uparrow_{n}$. From $\phi(I)=\lim _{n \rightarrow \infty}\left\|H_{n} x\right\|^{2}$, it follows that the sequence of real numbers $\left\{\left\|H_{n} x\right\|^{2}\right\}$ is a Cauchy sequence. We now show that the sequence $\left\{H_{n} x\right\}$ is a Cauchy sequence in $\mathscr{H}$. In fact, for $n \geqq m$, 


$$
\begin{aligned}
\left\|H_{n} x-H_{m} x\right\|^{2} & =\left\|H_{n} x\right\|^{2}+\left\|H_{m} x\right\|^{2}-2\left(H_{n} x, H_{m} x\right) \\
& \leqq\left\|H_{n} x\right\|^{2}+\left\|H_{m} x\right\|^{2}-2\left(H_{m} x, H_{m} x\right) \\
& =\left\|H_{n} x-H_{m} x\right\|^{2} \\
& \rightarrow 0 \text { as } n, m \rightarrow \infty .
\end{aligned}
$$

Let $y=\lim _{n \rightarrow \infty} H_{n} x$. For each $T \in M$,

$$
\begin{aligned}
\phi(T) & =\lim _{n \rightarrow \infty}\left(T H_{n} x, H_{n} x\right)=(T y, y) \\
& =\omega_{y}(T)
\end{aligned}
$$

and the proof is complete.

\section{References}

[1] J. Dixmier, Les algèbres d'opérateurs dans l'espace hilbertien (Gauthier-Villars, Paris, 1957).

[2] W. A. J. Luxemburg and A. C. Zaanen, Riesz spaces (California Institute of Technology, 1969).

[3] W. A. J. Luxemburg and A. C. Zaanen, 'Notes on Banach function spaces', Proc. Acad. Sci, Amsterdam; Note VI, A66 (1963), 655-668; Note VII, A66 (1963), 669-681; Note VII A67 (1964), 104-119; Note IX, A67 (1964), 360-376; Note X, A67 (1964), 493-506; Note XI, A67 (1964), 507-518; Note XII, A67 (1964), 519-529.

[4] W. A. J. Luxemburg, 'Notes on Banach function spaces', Proc. Acad. Sci. Amsterdam; Note XIV, A68 (1965), 230-248; Note XV, A68 (1965), 416-446; Note XVI, A68 (1965), 646-667.

[5] W. A. J. Luxemburg, 'Is every integral normal?', Bull. Amer. Math. Soc. 73 (1967), 685-688.

[6] N. v. Sz. -Nagy, Spektraldarstellung linearer transformation des hilbertschen raumes (Ergebnisse d. Math., Band 5, Springer, Berlin, 1942).

[7] R. Pallu de la Barrière, 'Sur les algèbres d'opérateurs dans les espaces hilbertiens', Bull. Soc. Math. Fr., 82 (1954), 1-51.

[8] R. J. Plymen, 'C*-algebras and Mackey's axioms', Comm. Math. Phys. 8 (1968), 132-146.

[9] S. Sakai, The theory of $W^{*}$-algebras (Yale Notes, 1962).

[10] B. Z. Vulikh, Introduction to the theory of partially ordered spaces (Wolters-Noordhoff, Groningen, 1967).

The Flinders University of South Australia

Bedford Park 5042

South Australia 